\title{
RESULTANT AND DISCRIMINANT OF ITERATE POLYNOMIALS
}

\author{
Eunmi ChOI
}

\begin{abstract}
The resultant and discriminant of composite polynomials were studied by McKay and Wang using some algebraic properties. In this paper we study the resultant and discriminant of iterate polynomials. We shall use elementary computations of matrices and block matrix determinants; this could provide not only the values but also the visual structure of resultant and discriminant from elementary matrix calculation.
\end{abstract}

\section{Introduction}

Let $f(x)$ be a polynomial in $K[x]$. We denote $f_{n}(x)=(f \circ f \circ \cdots \circ f)(x)$ the $n$-th iterate of $f$. It was studied the situations that the irreducibility and the separability of $f$ are preserved through all iteration $f_{n}$ for every $n$ ([8], [9]). The question whether $f_{n}$ is factored out into irreducible polynomials over a field $K$ is important in determining the Galois group $\operatorname{Gal}\left(f_{n} / K\right)$. One of the classical algebraic tools for determining irreducibility is the discriminant and resultant. The discriminant yields information that not only the roots of $f$ is repeated but also the roots are real or complex, and ration or irrational. Hence it gives a criterion whether the roots are in the field $K$, or are in an extension field, so we may have the structure of the Galois group [12, p.129]. Recently

Received June 1, 2010. Accepted September 14, 2010.

Mathematics Subject Classification : 11C20, 11R29, 13P15

Key Words: Resultant, Discriminant, Iterated polynomial

Supported by Hannam University Research Fund 2010 
resultants are applied to provide constructive solutions to problems in computer graphic [5], robotic [6], geometric modeling [3] and algorithmic algebraic geometry [2].

In this paper we study the resultant and discriminant of composite and iterate polynomials systematically. It was McKay and Wang [7] who studied resultant and discriminant of composite of two polynomials. While they used algebraic properties of resultants for those calculations, we shall use elementary computations of matrices and block matrix determinants.

\section{Preliminaries}

Let $f(x)=\sum_{i=0}^{n} a_{i} x^{i}$ and $g(x)=\sum_{j=0}^{m} b_{j} x^{j}$ be two polynomials over a field $K$. Let $\alpha_{i}(i=1, \cdots, n)$ be roots of $f(x)$ and $\beta_{j}(j=1, \cdots, m)$ be roots of $g(x)$ in some splitting fields over $K$.

Definition. The resultant of $f(x)$ and $g(x)$ is defined by

$$
R(f(x), g(x))=R(f, g)=a_{n}^{m} b_{m}^{n} \prod_{i=1}^{n} \prod_{j=1}^{m}\left(\alpha_{i}-\beta_{j}\right),
$$

while the discriminant of $f$ is defined by

$$
\Delta(f)=a_{n}^{2 n-2} \prod_{1 \leq i<j \leq n}\left(\alpha_{i}-\alpha_{j}\right)^{2} .
$$

The well known discriminant of the quadratic $f(x)=a x^{2}+b x+c$ comes from that $f(x)$ has two roots $\left(-b \pm \sqrt{b^{2}-4 a c}\right) / 2 a$ so that $\Delta(f)=$ $b^{2}-4 a c$. May refer to [1] and [11] as standard references for resultant and discriminants.

Lemma 1. Assume the same context as above. Then

(1) $R(f, g)=a_{n}^{m} \prod_{i=1}^{n} g\left(\alpha_{i}\right)=(-1)^{n m} b_{m}^{n} \prod_{j=1}^{m} f\left(\beta_{j}\right)=(-1)^{n m} R(g, f)$. 
(2) $R(f, g)=\prod_{i, j}\left(\alpha_{i}-\beta_{j}\right)$ if $f, g$ are monic.

(3) $R(x-a, g)=b_{m}\left(a-\beta_{1}\right) \cdots\left(a-\beta_{m}\right)=g(a)$ for any $a \in K$.

Owing to the roots $\alpha_{i}$ of $f(x)$ and $\beta_{j}$ of $g(x)$, if we write $f(x)=$ $a_{n} \prod_{i=1}^{n}\left(x-\alpha_{i}\right)$ and $g(x)=b_{m} \prod_{j=1}^{m}\left(x-\beta_{j}\right)$ then Lemma 1 follows immediately that

$$
\begin{aligned}
R(f, g) & =a_{n}^{m} \prod_{i=1}^{n}\left(b_{m} \prod_{j=1}^{m}\left(\alpha_{i}-\beta_{j}\right)\right)=a_{n}^{m} \prod_{i=1}^{n} g\left(\alpha_{i}\right) \\
& =b_{m}^{n}(-1)^{n m} \prod_{j=1}^{m} a_{n}^{m} \prod_{i=1}^{n}\left(\beta_{j}-\alpha_{i}\right)=(-1)^{n m} b_{m}^{n} \prod_{j=1}^{m} f\left(\beta_{j}\right) .
\end{aligned}
$$

Lemma 2. Let $f(x)$ be with leading coefficient $a_{n}$, and $f^{\prime}$ be the formal derivative of $f$. Then $\Delta(f)=(-1)^{n(n-1) / 2} a_{n}^{-1} R\left(f, f^{\prime}\right)$. Moreover for any $f_{i}(x), g_{j}(x)$,

$$
\begin{gathered}
R\left(\prod_{i=1}^{u} f_{i}, \prod_{j=1}^{v} g_{j}\right)=\prod_{i, j=1}^{u, v} R\left(f_{i}, g_{j}\right), \\
\Delta\left(\prod_{i=1}^{u} f_{i}\right)=\prod_{i=1}^{u} \Delta\left(f_{i}\right)\left(\prod_{1 \leq i<j \leq u} R\left(f_{i}, f_{j}\right)\right)^{2} .
\end{gathered}
$$

One of the most remarkable results about resultant is that $R(f, g)$ is the determinant of the Sylvester matrix of $f$ and $g$.

Lemma 3. Let $f(x)=\sum_{i=0}^{n} a_{i} x^{i}$ and $g(x)=\sum_{j=0}^{m} b_{j} x^{j} \in K[x]$. Then $R(f, g)$ is the determinant of the $(n+m) \times(n+m)$ Sylvester 
matrix:

$$
\left[\begin{array}{cccccccccc}
a_{n} & a_{n-1} & a_{n-2} & \cdots & \cdots & a_{1} & a_{0} & 0 & \cdots & 0 \\
0 & a_{n} & a_{n-1} & \cdots & \cdots & a_{2} & a_{1} & a_{0} & \cdots & 0 \\
\vdots & \vdots & \ddots & \cdots & \cdots & \vdots & \vdots & \vdots & \ddots & \vdots \\
0 & \cdots & 0 & a_{n} & a_{n-1} & a_{n-2} & \cdots & \cdots & \cdots & a_{0} \\
b_{m} & b_{m-1} & b_{m-2} & \cdots & b_{1} & b_{0} & 0 & 0 & \cdots & \cdots \\
0 & b_{m} & b_{m-1} & \cdots & b_{2} & b_{1} & b_{0} & 0 & \cdots & 0 \\
\vdots & \vdots & \ddots & \cdots & \cdots & \vdots & \vdots & \vdots & \ddots & \vdots \\
0 & 0 & \cdots & 0 & 0 & b_{m} & b_{m-1} & b_{m-2} & \cdots & b_{0}
\end{array}\right]
$$

Thus many properties of resultant as well as discriminant follow from the Sylvester matrix without calculating roots explicitly.

\section{Resultant and Discriminant of the composition of Poly- nomials}

The resultant of composite polynomials was investigated by McKay and Wang under the name of Chain rule.

Lemma 4. [7] Let $f(x), g(x)$ and $h(x)$ be of degree $n, m$ and $t$ respectively. If $h(x)$ has leading coefficient $c_{t}$ then $R(f \circ h, g \circ h)=$ $\left[c_{t}^{m n} R(f, g)\right]^{t}$.

In the proof of [7, Theorem 6], all possible situations were divided into 5 cases; here we recall one of the cases that $\operatorname{deg} h=t \geq 1$. If $f(x)=$ $a_{n} \prod_{i=1}^{n}\left(x-\alpha_{i}\right), g(x)=b_{m} \prod_{j=1}^{m}\left(x-\beta_{j}\right)$ then $f \circ h=a_{n} \prod_{i=1}^{n}\left(h(x)-\alpha_{i}\right)$ and $g \circ h=b_{m} \prod_{j=1}^{m}\left(h(x)-\beta_{j}\right)$ with $\operatorname{deg}\left(h(x)-\alpha_{i}\right)=\operatorname{deg}\left(h(x)-\beta_{j}\right)=t$ for all $i, j$. By Lemma 1 and 2, we have

$$
\begin{aligned}
& R(f \circ h, g \circ h)=R\left(a_{n} \prod_{i=1}^{n}\left(h(x)-\alpha_{i}\right), b_{m} \prod_{j=1}^{m}\left(h(x)-\beta_{j}\right)\right) \\
= & \prod_{j=1}^{m} R\left(a_{n}, h(x)-\beta_{j}\right) \prod_{i=1}^{n} R\left(h(x)-\alpha_{i}, b_{m}\right) \prod_{i=1}^{n} \prod_{j=1}^{m} R\left(h(x)-\alpha_{i}, h(x)\right.
\end{aligned}
$$




$$
\begin{aligned}
& =\prod_{j=1}^{\left.-\beta_{j}\right)} a_{n}^{t} \prod_{i=1}^{n} b_{m}^{t} \prod_{i=1}^{n} \prod_{j=1}^{m} c_{t}^{t} R\left(h(x)-\alpha_{i}, \alpha_{i}-\beta_{j}\right) \\
& =a_{n}^{m t} b_{m}^{n t} \prod_{i=1}^{n} \prod_{j=1}^{m} c_{t}^{t}\left(\alpha_{i}-\beta_{j}\right)^{t}=\left[c_{t}^{m n} R(f, g)\right]^{t} .
\end{aligned}
$$

This proof makes use of the roots of the given polynomials, however it is not easy to find all solutions. We shall use only basic definition of resultant in terms of matrix and its determinant to get resultant of polynomials. It could provide an advantage to see them visually. We begin with a simple case.

Theorem 5. Let $f(x)=a_{3} x^{3}+a_{2} x^{2}+a_{1} x+a_{0}$ and $g(x)=b_{2} x^{2}+$ $b_{1} x+b_{0}$. Then $R\left(f\left(c x^{2}\right), g\left(c x^{2}\right)\right)=\left(c^{6} R(f, g)\right)^{2} \quad$ for $c \in K$.

Proof. $R\left(f\left(c x^{2}\right), g\left(c x^{2}\right)\right)$ is the determinant of the $10 \times 10$ matrix:

$$
\begin{aligned}
& R\left(f\left(c x^{2}\right), g\left(c x^{2}\right)\right) \\
& =\left|\begin{array}{cccccccccc}
a_{3} c^{3} & 0 & a_{2} c^{2} & 0 & a_{1} c & 0 & a_{0} & 0 & 0 & 0 \\
0 & a_{3} c^{3} & 0 & a_{2} c^{2} & 0 & a_{1} c & 0 & a_{0} & 0 & 0 \\
0 & 0 & a_{3} c^{3} & 0 & a_{2} c^{2} & 0 & a_{1} c & 0 & a_{0} & 0 \\
0 & 0 & 0 & a_{3} c^{3} & 0 & a_{2} c^{2} & 0 & a_{1} c & 0 & a_{0} \\
b_{2} c^{2} & 0 & b_{1} c & 0 & b_{0} & 0 & 0 & 0 & 0 & 0 \\
0 & b_{2} c^{2} & 0 & b_{1} c & 0 & b_{0} & 0 & 0 & 0 & 0 \\
0 & 0 & b_{2} c^{2} & 0 & b_{1} c & 0 & b_{0} & 0 & 0 & 0 \\
0 & 0 & 0 & b_{2} c^{2} & 0 & b_{1} c & 0 & b_{0} & 0 & 0 \\
0 & 0 & 0 & 0 & b_{2} c^{2} & 0 & b_{1} c & 0 & b_{0} & 0 \\
0 & 0 & 0 & 0 & 0 & b_{2} c^{2} & 0 & b_{1} c & 0 & b_{0}
\end{array}\right|
\end{aligned}
$$

With the $2 \times 2$ identity matrix $I_{2}, R\left(f\left(c x^{2}\right), g\left(c x^{2}\right)\right)$ is the $5 \times 5$ block matrix:

$$
R\left(f\left(c x^{2}\right), g\left(c x^{2}\right)\right)=\left|\begin{array}{ccccc}
a_{3} c^{3} I_{2} & a_{2} c^{2} I_{2} & a_{1} c I_{2} & a_{0} I_{2} & 0 \\
0 & a_{3} c^{3} I_{2} & a_{2} c^{2} I_{2} & a_{1} c I_{2} & a_{0} I_{2} \\
b_{2} c^{2} I_{2} & b_{1} c I_{2} & b_{0} I_{2} & 0 & 0 \\
0 & b_{2} c^{2} I_{2} & b_{1} c I_{2} & b_{0} I_{2} & 0 \\
0 & 0 & b_{2} c^{2} I_{2} & b_{1} c I_{2} & b_{0} I_{2}
\end{array}\right|
$$


Then by taking out $c^{3}, c^{2}, c$ and $\frac{1}{c}$ from $1,2,3$, and 5 th column we have

$$
\begin{aligned}
& R\left(f\left(c x^{2}\right), g\left(c x^{2}\right)\right)=\left(c^{3} c^{2} c \frac{1}{c}\right)^{2}\left|\begin{array}{ccccc}
a_{3} I_{2} & a_{2} I_{2} & a_{1} I_{2} & a_{0} I_{2} & 0 \\
0 & a_{3} c I_{2} & a_{2} c I_{2} & a_{1} c I_{2} & a_{0} c I_{2} \\
b_{2} \frac{1}{c} I_{2} & b_{1} \frac{1}{c} I_{2} & b_{0} \frac{1}{c} I_{2} & 0 & 0 \\
0 & b_{2} I_{2} & b_{1} I_{2} & b_{0} I_{2} & 0 \\
0 & 0 & b_{2} c I_{2} & b_{1} c I_{2} & b_{0} c I_{2}
\end{array}\right| \\
& =\left(c^{6}\right)^{2}\left|\begin{array}{ccccc}
a_{3} I_{2} & a_{2} I_{2} & a_{1} I_{2} & a_{0} I_{2} & 0 \\
0 & a_{3} I_{2} & a_{2} I_{2} & a_{1} I_{2} & a_{0} I_{2} \\
b_{2} I_{2} & b_{1} I_{2} & b_{0} I_{2} & 0 & 0 \\
0 & b_{2} I_{2} & b_{1} I_{2} & b_{0} I_{2} & 0 \\
0 & 0 & b_{2} I_{2} & b_{1} I_{2} & b_{0} I_{2}
\end{array}\right|=\left(c^{6}\right)^{2}\left|R(f, g) I_{2}\right| \\
& =\left(c^{6} R(f, g)\right)^{2}
\end{aligned}
$$

We can generalize the above.

Theorem 6. Let $f(x)=\sum_{i=0}^{n} a_{i} x_{i}$ and $g(x)=\sum_{j=0}^{m} b_{j} x^{j}$. Then $R(f \circ h, g \circ h)=\left[c^{n m} R(f, g)\right]^{t}$ for $h(x)=c x^{t}$.

Proof. We first assume $c=1$. Since $f(h(x))$ and $g(h(x))$ are of degree $n t$ and $m t, R(f \circ h, g \circ h)$ is the determinant of the $(n+m) t \times$ $(n+m) t$ matrix:

$$
R(f \circ h, g \circ h)=R\left(f\left(x^{t}\right), g\left(x^{t}\right)\right)
$$




$$
=\left|\begin{array}{ccccccccccc}
a_{n} & 0 & \cdots & 0 & a_{n-1} & 0 & \cdots & 0 & a_{n-2} & 0 & \cdots \\
0 & a_{n} & \cdots & 0 & 0 & a_{n-1} & \cdots & 0 & 0 & a_{n-2} & \cdots \\
0 & 0 & \ddots & a_{n} & 0 & 0 & \ddots & a_{n-1} & 0 & 0 & \ddots \\
& & & & & & & & & & \\
0 & 0 & \cdots & 0 & a_{n} & 0 & \cdots & 0 & a_{n-1} & 0 & \cdots \\
0 & 0 & \cdots & 0 & 0 & a_{n} & \cdots & 0 & 0 & a_{n-1} & \cdots \\
\cdots & \cdots & \cdots & \cdots & \cdots & \cdots & \ddots & \vdots & \cdots & \cdots & \cdots \\
b_{m} & 0 & \cdots & 0 & b_{m-1} & 0 & \cdots & 0 & b_{m-2} & 0 & \cdots \\
0 & b_{m} & \cdots & 0 & 0 & b_{m-1} & \cdots & 0 & 0 & b_{m-2} & \cdots \\
0 & 0 & \ddots & b_{m} & 0 & 0 & \ddots & b_{m-1} & 0 & 0 & \ddots \\
0 & 0 & \cdots & 0 & b_{m} & 0 & \cdots & 0 & b_{m-1} & 0 & \cdots \\
\vdots & \vdots & \cdots & \vdots & \vdots & \vdots & \ddots & \vdots & \vdots & \vdots & \cdots
\end{array}\right| .
$$

It consists of $n+m$ block matrices of size $t \times t$, so using the identity matrix $I_{t}$,

$$
R\left(f\left(x^{t}\right), g\left(x^{t}\right)\right)=\left|\begin{array}{ccccc}
a_{n} I_{t} & a_{n-1} I_{t} & a_{n-2} I_{t} & \ldots & \ldots \\
0 & a_{n} I_{t} & a_{n-1} I_{t} & a_{n-2} I_{t} & \ldots \\
\ldots & \ddots & \ldots & \ldots & \ldots \\
0 & \ldots & 0 & a_{n} I_{t} & \ldots a_{0} I_{t} \\
b_{m} I_{t} & b_{m-1} I_{t} & b_{m-2} I_{t} & \ldots & \ldots \\
0 & b_{m} I_{t} & b_{m-1} I_{t} & b_{m-2} I_{t} & \ldots \\
\ldots & \ddots & \ldots & \ldots & \ldots \\
0 & \ldots & b_{m} I_{t} & b_{m-1} I_{t} & \ldots b_{0} I_{t}
\end{array}\right| .
$$

where this is the determinant of $(n+m) \times(n+m)$ matrix. Thus we have

$$
R(f \circ h, g \circ h)=R\left(f\left(x^{t}\right), g\left(x^{t}\right)\right)=\left|R(f, g) I_{t \times t}\right|=R(f, g)^{t} .
$$

Now for any $c$, we have $R(f \circ h, g \circ h)=\left(c^{n m} R(f, g)\right)^{t}$ by extracting common $c$ 's from each columns.

Lemma 7. [10] Set $M=\left[\begin{array}{ll}A & B \\ C & D\end{array}\right]$ with the same size square blocks $A, B, C, D$. If either $B=0$ or $C=0$ then $|M|=|A||D|$. Moreover 
$|M|=|A D-B C|$ if either at least one of the blocks $A, B, C, D$ is equal to 0 , or $C D=D C$.

Theorem 8. Let $\operatorname{deg} f=\operatorname{deg} g=2$ and $h(x)=c x^{t}+k$ be a binomial. Then

$$
R(f \circ h, g \circ h)=\left[c^{4} R(f, g)\right] .
$$

Proof. Let $F=f \circ h$ and $G=g \circ h$. We first assume $t=1$ and $c=1$. Then

$$
\begin{aligned}
& =\left|\begin{array}{cccc}
R(F, G) & \multicolumn{3}{c}{b_{2}} \\
a_{2} & 0 & 0 \\
2 k a_{2}+a_{1} & a_{2} & 2 k b_{2}+b_{1} & b_{2} \\
k^{2} a_{2}+k a_{1}+a_{0} & 2 k a_{2}+a_{1} & k^{2} b_{2}+k b_{1}+b_{0} & 2 k b_{2}+b_{1} \\
0 & k^{2} a_{2}+k a_{1}+a_{0} & 0 & k^{2} b_{2}+k b_{1} \\
& & & +b_{0}
\end{array}\right| \\
& =-\left|\begin{array}{cccc}
a_{2} & b_{2} & 0 & 0 \\
a_{1} & b_{1} & a_{2} & b_{2} \\
a_{0} & b_{0} & a_{1} & b_{1} \\
0 & 0 & k^{2} a_{2}+k a_{1}+a_{0} & k^{2} b_{2}+k b_{1}+b_{0}
\end{array}\right| \\
& -\left|\begin{array}{cccc}
a_{2} & b_{2} & 0 & 0 \\
a_{1} & b_{1} & a_{2} & b_{2} \\
0 & 0 & k a_{2} & k b_{2} \\
0 & 0 & k^{2} a_{2}+k a_{1}+a_{0} & k^{2} b_{2}+k b_{1}+b_{0}
\end{array}\right| \\
& =-\left|\begin{array}{cccc}
a_{2} & b_{2} & 0 & 0 \\
a_{1} & b_{1} & a_{2} & b_{2} \\
a_{0} & b_{0} & a_{1} & b_{1} \\
0 & 0 & a_{0} & b_{0}
\end{array}\right|-k\left|\begin{array}{cccc}
a_{2} & b_{2} & 0 & 0 \\
a_{1} & b_{1} & a_{2} & b_{2} \\
a_{0} & b_{0} & a_{1} & b_{1} \\
0 & 0 & k a_{2}+a_{1} & k b_{2}+b_{1}
\end{array}\right| \\
& -k\left|\begin{array}{cccc}
a_{2} & b_{2} & 0 & 0 \\
a_{1} & b_{1} & 0 & 0 \\
0 & 0 & a_{2} & b_{2} \\
0 & 0 & k a_{1}+a_{0} & k b_{1}+b_{0}
\end{array}\right| .
\end{aligned}
$$


Due to Lemma 7, we have

$$
\begin{aligned}
& \left|\begin{array}{cccc}
a_{2} & b_{2} & 0 & 0 \\
a_{1} & b_{1} & a_{2} & b_{2} \\
a_{0} & b_{0} & a_{1} & b_{1} \\
0 & 0 & k a_{2}+a_{1} & k b_{2}+b_{1}
\end{array}\right|=\left|\begin{array}{cccc}
a_{2} & b_{2} & 0 & 0 \\
a_{1} & b_{1} & a_{2} & b_{2} \\
a_{0} & b_{0} & -k a_{2} & -k b_{2} \\
0 & 0 & k a_{2}+a_{1} & k b_{2}+b_{1}
\end{array}\right| \\
& =-\left|\begin{array}{cccc}
a_{2} & b_{2} & 0 & 0 \\
k a_{1}+a_{0} & k b_{1}+b_{0} & 0 & 0 \\
a_{1} & b_{1} & a_{2} & b_{2} \\
0 & 0 & k a_{2}+a_{1} & k b_{2}+b_{1}
\end{array}\right| \\
& =-\left|\begin{array}{ccc}
a_{2} & b_{2} \\
k a_{1}+a_{0} & k b_{1}+b_{0}
\end{array}\right|\left|\begin{array}{cc}
a_{2} & b_{2} \\
a_{1} & b_{1}
\end{array}\right|
\end{aligned}
$$

Hence $R(f \circ h, g \circ h)=R(F, G)=-\left|\begin{array}{cccc}a_{2} & b_{2} & 0 & 0 \\ a_{1} & b_{1} & a_{2} & b_{2} \\ a_{0} & b_{0} & a_{1} & b_{1} \\ 0 & 0 & a_{0} & b_{0}\end{array}\right|=R(f, g)$.

When $c>1, R(f \circ h, g \circ h)=c^{4} R(f, g)$ by taking out $c$ in each column.

Now if $h(x)=x^{t}+k(t \geq 1)$ then $R(F, G)$ is the determinant of $4 t \times 4 t$ matrix that can be represented as block matrix in terms of the $t \times t$ identity matrix $I$ :

$$
\begin{aligned}
& R(F, G) \\
& =\left|\begin{array}{cccc}
a_{2} I & 0 & b_{2} I & 0 \\
\left(2 k a_{2}+a_{1}\right) I & a_{2} I & \left(2 k b_{2}+b_{1}\right) I & b_{2} I \\
\left(k^{2} a_{2}+k a_{1}+a_{0}\right) I & \left(2 k a_{2}+a_{1}\right) I & \left(k^{2} b_{2}+k b_{1}+b_{0}\right) I & \left(2 k b_{2}+b_{1}\right) I \\
0 & \left(k^{2} a_{2}+k a_{1}+a_{0}\right) I & 0 & \left(k^{2} b_{2}+k b_{1}+b_{0}\right) I
\end{array}\right| \\
& =|R(f, g) I|=R(f, g)^{t} \text {. }
\end{aligned}
$$

Therefore for any $h(x)=c x^{t}+k$ with $t \geq 1$, we have $R(F, G)=$ $\left(c^{4} R(f, g)\right)^{t}$.

This corresponds to Lemma 4. 
Theorem 9. If $f(x)$ is of degree $n$ with leading coefficient $a_{n}$, and $h(x)=c x^{t}$ then

$$
\Delta(f \circ h)=(-1)^{n t(n t+n-2) / 2} a_{n}^{t-1} c^{n(n t-1)} t^{n t} f(0)^{t-1} \Delta(f)^{t} .
$$

Proof. Since $(f \circ h)(x)=f\left(c x^{t}\right)$ is of degree $n t$ with leading coefficient $a c^{n}$,

$$
\begin{aligned}
\Delta( & f \circ h)=(-1)^{n t(n t-1) / 2}\left(a_{n} c^{n}\right)^{-1} R\left(f\left(c x^{t}\right),\left(f\left(c x^{t}\right)\right)^{\prime}\right) \\
= & (-1)^{n t(n t-1) / 2}\left(a_{n} c^{n}\right)^{-1} R\left(f\left(c x^{t}\right), f^{\prime}\left(c x^{t}\right) c t x^{t-1}\right) \\
= & (-1)^{n t(n t-1) / 2}\left(a_{n} c^{n}\right)^{-1} R\left(f\left(c x^{t}\right), c t\right) R\left(f\left(c x^{t}\right), x^{t-1}\right) R\left(f\left(c x^{t}\right),\right. \\
& \left.f^{\prime}\left(c x^{t}\right)\right)
\end{aligned}
$$

by Lemma 2. Due to Lemma $1, R\left(f\left(c x^{t}\right), c t\right)=(c t)^{\operatorname{deg}\left(f\left(c x^{t}\right)\right)}=(c t)^{n t}$, and $R\left(f\left(c x^{t}\right), x^{t-1}\right)=f(0)^{t-1}$ since 0 is the only root of $x^{t-1}$. Moreover we have

$$
\begin{aligned}
R\left(f\left(c x^{t}\right), f^{\prime}\left(c x^{t}\right)\right) & =R\left(f \circ h, f^{\prime} \circ h\right) \\
& =\left(c^{n(n-1)} R\left(f, f^{\prime}\right)\right)^{t} \\
& =\left(c^{n(n-1)}(-1)^{n(n-1) / 2} a_{n} \Delta(f)\right)^{t} .
\end{aligned}
$$

Hence it follows immediately that

$$
\begin{aligned}
& \Delta(f \circ h) \\
= & (-1)^{n t(n t-1) / 2}\left(a_{n} c^{n}\right)^{-1}(c t)^{n t} f(0)^{t-1}(-1)^{n t(n-1) / 2} c^{n t(n-1)} a^{t} \Delta(f)^{t} \\
= & (-1)^{n t(n t+n-2) / 2} a_{n}^{t-1} c^{n(n t-1)} t^{n t} f(0)^{t-1} \Delta(f)^{t} .
\end{aligned}
$$

We can generalize this to any polynomial $h(x)$.

Theorem 10. Let $f(x)$ and $h(x)$ be polynomials of degree $n$ and $t$ with leading coefficient $a_{n}$ and $c_{t}$. Then

$$
\Delta(f \circ h)=(-1)^{n t(n t+n-2) / 2} a_{n}^{t-1} c_{t}^{n(n t-t-1)} R\left(f \circ h, h^{\prime}\right) \Delta(f)^{t} .
$$


Moreover if we let $p(x)=\prod_{j=1}^{t-1}\left(x-h\left(\mu_{j}\right)\right)$ with roots $\mu_{j}$ of $h^{\prime}(x)$, then

$$
\Delta(f \circ h)=(-1)^{n\left(n t^{2}+n t-2\right) / 2} t^{n t} a_{n}^{t-1} c_{t}^{n(n t-1)} R(f, p) \Delta(f)^{t} .
$$

Proof. Similar to Theorem 9, we have

$$
\begin{aligned}
& \Delta(f \circ h)=(-1)^{n t(n t-1) / 2}\left(a_{n} c_{t}^{n}\right)^{-1} R\left(f \circ h,(f \circ h)^{\prime}\right) \\
& =(-1)^{n t(n t-1) / 2}\left(a_{n} c_{t}^{n}\right)^{-1} R\left(f \circ h, h^{\prime}\right) R\left(f \circ h, f^{\prime} \circ h\right) \\
& =(-1)^{n t(n t-1) / 2}\left(a_{n} c_{t}^{n}\right)^{-1} R\left(f \circ h, h^{\prime}\right)\left(c_{t}^{n(n-1)} R\left(f, f^{\prime}\right)\right)^{t} \\
& =(-1)^{n t(n t-1) / 2}\left(a_{n} c_{t}^{n}\right)^{-1} c_{t}^{n t(n-1)} R\left(f \circ h, h^{\prime}\right)\left((-1)^{n(n-1) / 2} a_{n} \Delta(f)\right)^{t} \\
& =(-1)^{n t(n t+n-2) / 2} a_{n}^{t-1} c_{t}^{n(n t-t-1)} R\left(f \circ h, h^{\prime}\right) \Delta(f)^{t} .
\end{aligned}
$$

We may write $h^{\prime}(x)=t c_{t} \prod_{j=1}^{t-1}\left(x-\mu_{j}\right)$ for roots $\mu_{j}$ of $h^{\prime}(x)$ because $h(x)$ is of degree $t$ with leading coefficient $c_{t}$. Then with $f(x)=a_{n} \prod_{i=1}^{n}(x-$ $\alpha_{i}$ ), we have

$$
\begin{aligned}
& R\left(f \circ h, h^{\prime}\right)=R\left(a_{n} \prod_{i=1}^{n}\left(h(x)-\alpha_{i}\right), t c_{t} \prod_{j=1}^{t-1}\left(x-\mu_{j}\right)\right) \\
& =\prod_{j=1}^{t-1} R\left(a_{n}, x-\mu_{j}\right) \prod_{i=1}^{n} R\left(h(x)-\alpha_{i}, t c_{t}\right) \prod_{i=1}^{n} \prod_{j=1}^{t-1} R\left(h(x)-\alpha_{i}, x-\mu_{j}\right) \\
& =a_{n}^{t-1}\left(t c_{t}\right)^{n t} \prod_{i=1}^{n} \prod_{j=1}^{t-1}(-1)^{t}\left(h\left(\mu_{j}\right)-\alpha_{i}\right) \\
& =a_{n}^{t-1}\left(t c_{t}\right)^{n t}(-1)^{n(t-1)} \prod_{i=1}^{n} \prod_{j=1}^{t-1}\left(\alpha_{i}-h\left(\mu_{j}\right)\right)
\end{aligned}
$$

for $t(t-1)$ is even. On the other hand since

$$
R(f, p)=R\left(a_{n} \prod_{i=1}^{n}\left(x-\alpha_{i}\right), \prod_{j=1}^{t-1}\left(x-h\left(\mu_{j}\right)\right)=a_{n}^{t-1} \prod_{i=1}^{n} \prod_{j=1}^{t-1}\left(\alpha_{i}-h\left(\mu_{j}\right)\right)\right.
$$

with $p(x)=\prod_{j=1}^{t-1}\left(x-h\left(\mu_{j}\right)\right)$, we have

$$
R\left(f \circ h, h^{\prime}\right)=(-1)^{n(t-1)}\left(t c_{t}\right)^{n t} R(f, p),
$$


thus

$$
\begin{aligned}
\Delta(f \circ h) & =(-1)^{n t(n t+n-2) / 2}(-1)^{n(t-1)} t^{n t} a_{n}^{t-1} c_{t}^{n(n t-t-1)} c_{t}^{n t} R(f, p) \Delta(f)^{t} \\
& =(-1)^{n\left(n t^{2}+n t-2\right) / 2} t^{n t} a_{n}^{t-1} c_{t}^{n(n t-1)} R(f, p) \Delta(f)^{t} .
\end{aligned}
$$

For example if $h(x)=c x^{t}$ then $h^{\prime}(x)=t c x^{t-1}$ and

$R\left(f \circ h, h^{\prime}\right)=R\left(a_{n}, x^{t-1}\right) \prod_{i=1}^{n} R\left(h(x)-\alpha_{i}, t c\right) \prod_{i=1}^{n} R\left(h(x)-\alpha_{i}, x^{t-1}\right)$ $=a_{n}^{t-1}(t c)^{n t} \prod_{i=1}^{n}\left(h(0)-\alpha_{i}\right)^{t-1}=(-1)^{t(t-1)} t^{n t} c^{n t}\left(a_{n} \prod_{i=1}^{n}\left(\alpha_{i}\right)\right)^{t-1}=$ $t^{n t} c^{n t} f(0)^{t-1}$

since $f(0)=a_{n} \prod_{i=1}^{n}\left(-\alpha_{i}\right)$ and $t(t-1)$ even. Therefore

$$
\begin{aligned}
\Delta(f \circ h) & =(-1)^{n t(n t+n-2) / 2} a_{n}^{t-1} c_{t}^{n(n t-t-1)} R\left(f \circ h, h^{\prime}\right) \Delta(f)^{t} \\
& =(-1)^{n t(n t+n-2) / 2} a_{n}^{t-1} c_{t}^{n(n t-1)} t^{n t} f(0)^{t-1} \Delta(f)^{t},
\end{aligned}
$$

this corresponds to Theorem 9 .

\section{Resultant and Discriminant of iterate polynomials}

We shall study resultant and discriminant of the composite polynomial of itself; the iterate $f \circ \cdots \circ f=f_{n}(x)$. Clearly $f(x)=f_{1}(x)$ and $\operatorname{deg} f_{n}(x)=(\operatorname{deg} f(x))^{n}$.

Theorem 11. Let $f(x)=a x+b$ be a linear polynomial in $K[x]$.

(1) $R\left(f_{n}, f_{n}^{\prime}\right)=a^{n}$ and $\Delta\left(f_{n}\right)=1$ for all $n \geq 1$.

(2) $R\left(f_{n}, f\right)=-a b\left(a^{n-2}+\cdots+a^{2}+a+1\right)$ for $n>1$, while $R\left(f_{1}, f\right)=0$.

(3) Moreover $R\left(f_{n}, f\right)=-a f_{n-1}(0)$ for all $n>1$.

Proof. Clearly $f_{2}(x)=a^{2} x+(a+1) b, f_{3}(x)=a^{3} x+\left(a^{2}+a+1\right) b, \cdots$, and

$$
f_{n}(x)=a^{n} x+\left(\sum_{i=0}^{n-1} a^{i}\right) b=a^{n} x+\frac{a^{n}-1}{a-1} b .
$$


(1) $R\left(f, f^{\prime}\right)=R(a x+b, a)=a$ and $R\left(f_{2}, f_{2}^{\prime}\right)=R\left(a^{2} x+(a+1) b, a^{2}\right)=a^{2}$.

So

$$
\begin{aligned}
R\left(f_{n}, f_{n}^{\prime}\right) & =R\left(a^{n} x+\sum_{i=0}^{n-1} a_{i} b, a^{n}\right)=a^{n} \text { and } \Delta\left(f_{n}\right)=a^{-n} R\left(f_{n}, f_{n}^{\prime}\right)=1 . \\
(2) R\left(f_{2}, f\right) & =\left|\begin{array}{cc}
a^{2} & (a+1) b \\
a & b
\end{array}\right|=a b\left|\begin{array}{cc}
a & a+1 \\
1 & 1
\end{array}\right|=a b\left|\begin{array}{cc}
0 & 1 \\
1 & 1
\end{array}\right|=-a b, \\
R\left(f_{3}, f\right) & =\left|\begin{array}{cc}
a^{3} & \left(a^{2}+a+1\right) b \\
a & b
\end{array}\right|=a b\left|\begin{array}{cc}
a^{2} & a^{2}+a+1 \\
1 & 1
\end{array}\right| \\
& =a b\left|\begin{array}{cc}
0 & a+1 \\
1 & 1
\end{array}\right|=-a b(a+1), \\
R\left(f_{4}, f\right) & =\left|\begin{array}{cc}
a^{4} & \left(a^{3}+a^{2}+a+1\right) b \\
a & b
\end{array}\right|=a b\left|\begin{array}{cc}
a^{3} & a^{3}+a^{2}+a+1 \\
1 & 1
\end{array}\right|=-a b\left(a^{2}+a+1\right) .
\end{aligned}
$$

Thus

$$
\begin{aligned}
R\left(f_{n}, f\right) & =\left|\begin{array}{cc}
a^{n} & \left(a^{n-1}+\cdots+a+1\right) b \\
a & b
\end{array}\right| \\
& =a b\left|\begin{array}{cc}
a^{n-1} & a^{n-1}+\cdots+a+1 \\
1 & 1
\end{array}\right| \\
& =a b\left|\begin{array}{cc}
0 & a^{n-2}+\cdots+a+1 \\
1 & 1
\end{array}\right|=-a b\left(a^{n-2}+\cdots+a+1\right) .
\end{aligned}
$$

(3) The constant terms of each iterate are

$f(0)=b, f_{2}(0)=f(b)=(a+1) b, f_{3}(0)=f((a+1) b)=\left(a^{2}+a+1\right) b, \cdots$, hence $f_{n}(0)=\left(\sum_{i=0}^{n-1} a^{i}\right) b$. Therefore

$$
R\left(f_{2}, f\right)=-a b=-a f_{1}(0), R\left(f_{3}, f\right)=-a b(a+1)=-a f_{2}(0),
$$

and $R\left(f_{4}, f\right)=-a b\left(a^{2}+a+1\right)=-a f_{3}(0)$, so $R\left(f_{n}, f\right)=-a f_{n-1}(0)$ for $n>1$.

We have more about resultant of iterate linear polynomial.

Theorem 12. Let $f(x)=a x+b$ be a linear polynomial. 
(1) $R\left(f_{n}, f_{k}\right)=-a^{k} b\left(a^{n-k-1}+\cdots+a+1\right)=-a^{k} f_{n-k}(0)$ for any $k<n$.

(2) $R\left(f_{n}, f_{k}\right)=R\left(f_{n}, f_{t}\right)+R\left(f_{t}, f_{k}\right)$ for any $k<t<n$.

Proof. Due to the Theorem 11, we have

$$
\begin{aligned}
R\left(f_{3}, f_{2}\right) & =\left|\begin{array}{cc}
a^{3} & \left(a^{2}+a+1\right) b \\
a^{2} & (a+1) b
\end{array}\right|=a^{2} b\left|\begin{array}{cc}
0 & 1 \\
1 & a+1
\end{array}\right|=-a^{2} b, \\
R\left(f_{4}, f_{3}\right) & =\left|\begin{array}{cc}
a^{4} & \left(a^{3}+a^{2}+a+1\right) b \\
a^{3} & \left(a^{2}+a+1\right) b
\end{array}\right|=a^{3} b\left|\begin{array}{cc}
0 & 1 \\
1 & a^{2}+a+1
\end{array}\right| \\
& =-a^{3} b .
\end{aligned}
$$

Similarly

$$
\begin{aligned}
R\left(f_{n}, f_{n-1}\right) & =\left|\begin{array}{cc}
a^{n} & \left(a^{n-1}+\cdots+a+1\right) b \\
a^{n-1} & \left(a^{n-2}+\cdots+a+1\right) b
\end{array}\right| \\
& =a^{n-1} b\left|\begin{array}{cc}
a & a^{n-1}+\cdots+1 \\
1 & a^{n-2}+\cdots+1
\end{array}\right|=a^{n-1} b . \\
& =a^{n-1} b\left|\begin{array}{cc}
0 & 1 \\
1 & a^{n-2}+\cdots+1
\end{array}\right|=-a^{n}
\end{aligned}
$$

It is also clear that

$$
\begin{aligned}
R\left(f_{4}, f_{2}\right) & =\left|\begin{array}{cc}
a^{4} & \left(a^{3}+a^{2}+a+1\right) b \\
a^{2} & (a+1) b
\end{array}\right|=a^{2} b\left|\begin{array}{cc}
0 & a+1 \\
1 & a+1
\end{array}\right|=-a^{2} b(a+1), \\
R\left(f_{5}, f_{3}\right) & =\left|\begin{array}{cc}
a^{5} & \left(a^{4}+\cdots+a+1\right) b \\
a^{3} & \left(a^{2}+a+1\right) b
\end{array}\right|=a^{3} b\left|\begin{array}{cc}
0 & a+1 \\
1 & a^{2}+a+1
\end{array}\right| \\
& =-a^{3} b(a+1),
\end{aligned}
$$

and analogously

$$
\begin{aligned}
R\left(f_{n}, f_{n-2}\right) & =\left|\begin{array}{cc}
a^{n} & \left(a^{n-1}+\cdots+a+1\right) b \\
a^{n-2} & \left(a^{n-3}+\cdots+a+1\right) b
\end{array}\right| \\
& =a^{n-2} b\left|\begin{array}{cc}
a^{2} & a^{n-1}+\cdots+1 \\
1 & a^{n-3}+\cdots+1
\end{array}\right| \\
& =a^{n-2} b\left|\begin{array}{cc}
0 & a+1 \\
1 & a^{n-3}+\cdots+1
\end{array}\right|=-a^{n-2} b(a+1) .
\end{aligned}
$$

It is not hard to see $R\left(f_{n}, f_{n-3}\right)=-a^{n-3} b\left(a^{2}+a+1\right)$. Thus for $k<n$,

$$
\begin{aligned}
R\left(f_{n}, f_{k}\right) & =\mid \begin{array}{cc}
a^{n} & \left(a^{n-1}+\cdots+a+1\right) b \\
a^{k} & \left(a^{k-1}+\cdots+a+1\right) b
\end{array} \\
& =a^{k} b\left|\begin{array}{cc}
a^{n-k} & a^{n-1}+\cdots+1 \\
1 & a^{k-1}+\cdots+1
\end{array}\right|
\end{aligned}
$$




$$
\begin{aligned}
& =a^{k} b\left|\begin{array}{cc}
0 & a^{n-k-1}+\cdots+1 \\
1 & a^{k-1}+\cdots+1
\end{array}\right| \\
& =-a^{k} b\left(a^{n-k-1}+\cdots+a+1\right) .
\end{aligned}
$$

(2) From the above calculation we have

$$
\begin{aligned}
R\left(f_{n}, f_{n-2}\right) & =-a^{n-2} b(a+1)=-a^{n-1} b-a^{n-2} b \\
& =R\left(f_{n}, f_{n-1}\right)+R\left(f_{n-1}, f_{n-2}\right) .
\end{aligned}
$$

Let $k<n$. If $a \neq 1$ then $R\left(f_{n}, f_{k}\right)=-a^{k} b \frac{1-a^{n-k}}{1-a}$ due to (1), thus

$$
\begin{aligned}
& R\left(f_{n}, f_{t}\right)+R\left(f_{t}, f_{k}\right)=-a^{t} b \frac{1-a^{n-t}}{1-a}-a^{k} b \frac{1-a^{t-k}}{1-a} \\
= & -a^{k} b\left(a^{t-k} \frac{1-a^{n-t}}{1-a}+\frac{1-a^{t-k}}{1-a}\right)=-a^{k} b \frac{1-a^{n-k}}{1-a}=R\left(f_{n}, f_{k}\right) .
\end{aligned}
$$

for any $k<t<n$. If $a=1$ then

$$
R\left(f_{n}, f_{t}\right)+R\left(f_{t}, f_{k}\right)=-b(n-t)-b(t-k)=-b(n-k)=R\left(f_{n}, f_{k}\right) .
$$

In particular $R\left(f_{n}, f_{n-2}\right)=R\left(f_{n}, f_{n-1}\right)+R\left(f_{n-1}, f_{n-2}\right)$. Theorem 11 and 12 yield the next corollary.

Corollary 13. Let $f(x)=a x+b$ be a linear polynomial.

(1) $R\left(f_{n}, f_{n-1}\right)=a R\left(f_{n-1}, f_{n-2}\right)=a^{2} R\left(f_{n-2}, f_{n-3}\right)=\cdots$

$$
=a^{n-2} R\left(f_{2}, f_{1}\right)=a^{n-2}\left(-a f_{1}(0)\right)=-a^{n-1} f_{1}(0)
$$

(2) $R\left(f_{n}, f_{n-2}\right)=a R\left(f_{n-1}, f_{n-3}\right)=a^{2} R\left(f_{n-2}, f_{n-4}\right)=\cdots$

$$
=a^{n-3} R\left(f_{3}, f_{1}\right)=a^{n-3}\left(-a f_{2}(0)\right)=-a^{n-2} f_{2}(0)
$$

(3) $R\left(f_{n}, f_{k}\right)=a R\left(f_{n-1}, f_{k-1}\right)=a^{2} R\left(f_{n-2}, f_{k-2}\right)=\cdots$

$$
=a^{k-1} R\left(f_{n-k+1}, f_{1}\right)=a^{k-1}\left(-a f_{n-k}(0)\right)=-a^{k} f_{n-k}(0)
$$

The above results correspond to Theorem 4 that

$$
R\left(f_{n}, f_{n-1}\right)=R\left(f_{n-1} \circ f, f_{n-2} \circ f\right)=a R\left(f_{n-1}, f_{n-2}\right) .
$$

In fact Theorem 4 can be used to get the same result as above. 
Corollary 14. If $f(x)=a x+b$ then $R\left(f_{n}, f\right)=-a b\left(\sum_{i=0}^{n-2} a^{i}\right)$. If $a=1$ then $R\left(f_{n}, f_{n}^{\prime}\right)=1=\Delta\left(f_{n}\right), R\left(f_{n}, f\right)=-n b$ and $R\left(f_{n}, f_{k}\right)=$ $-b(n-k)$ for $n>k$.

Proof. Let $I_{d}(x)=x$ be the identity map. Due to Theorem 4, we have

$$
\begin{gathered}
R\left(f_{2}, f\right)=R\left(f \circ f, I_{d} \circ f\right)=a R\left(f, I_{d}\right)=-a \prod_{I_{d}(\beta)=0} f(\beta)=-a f(0)=-a b, \\
R\left(f_{3}, f\right)=R\left(f_{2} \circ f, I_{d} \circ f\right)=a R\left(f_{2}, I_{d}\right)=-a \prod_{I_{d}(\beta)=0} f(\beta)=-a f_{2}(0) \\
=-a b(a+1) .
\end{gathered}
$$

Thus

$$
R\left(f_{n}, f\right)=R\left(f_{n-1} \circ f, I_{d} \circ f\right)=-a f_{n-1}(0)=-a b\left(\sum_{i=0}^{n-2} a^{i}\right) .
$$

If $f(x)=x+b$ then $f_{n}(x)=x+n b$, so $R\left(f_{n}, f_{n}^{\prime}\right)=1=\Delta\left(f_{n}\right)$. Thus

$$
R\left(f_{n}, f\right)=-n b \text { and } R\left(f_{n}, f_{k}\right)=-b(n-k), \quad n>k .
$$

Now for the discriminant of iterate quadratic polynomial according to resultants:

Theorem 15. If $f(x)=a x^{2}+b$ then $\Delta(f)=-2^{2} a b, \Delta\left(f_{2}\right)=$ $4^{4} a^{9} b^{3}(a b+1)$ and $\Delta\left(f_{3}\right)=8^{8} a^{49} b^{7}(a b+1)^{2}\left((a b)^{3}+2(a b)^{2}+a b+1\right)$.

Proof. It is clear that $R\left(f, f^{\prime}\right)=\left|\begin{array}{ccc}a & 0 & b \\ 2 a & 0 & 0 \\ 0 & 2 a & 0\end{array}\right|=2^{2} a^{2}\left|\begin{array}{ccc}a & 0 & b \\ 1 a & 0 & 0 \\ 0 & 1 a & 0\end{array}\right|=2^{2} a^{2} b\left|\begin{array}{cc}1 & 0 \\ 0 & 1\end{array}\right|=2^{2} a^{2} b$, thus $\Delta(f)=-2^{2} a b$. Moreover since $f_{2}(x)=a^{3} x^{4}+2 a^{2} b x^{2}+b(a b+1)$ we have 
$R\left(f_{2}, f_{2}^{\prime}\right)=\left|\begin{array}{lllllll}a^{3} & 0 & 2 a^{2} b & 0 & b(a b+1) & 0 & 0 \\ 0 & a^{3} & 0 & 2 a^{2} b & 0 & b(a b+1) & 0 \\ 0 & 0 & a^{3} & 0 & 2 a^{2} b & 0 & b(a b+1) \\ 4 a^{3} & 0 & 4 a^{2} b & 0 & 0 & 0 & 0 \\ 0 & 4 a^{3} & 0 & 4 a^{2} b & 0 & 0 & 0 \\ 0 & 0 & 4 a^{3} & 0 & 4 a^{2} b & 0 & 0 \\ 0 & 0 & 0 & 4 a^{3} & 0 & 4 a^{2} b & 0\end{array}\right|$

$=a^{10} b(a b+1)\left|\begin{array}{llllll}1 & 0 & 2 b & 0 & b(a b+1) & 0 \\ 0 & 1 & 0 & 2 b & 0 & b(a b+1) \\ 4 & 0 & 4 b & 0 & 0 & 0 \\ 0 & 4 & 0 & 4 b & 0 & 0 \\ 0 & 0 & 4 a & 0 & 4 a^{2} b & 0 \\ 0 & 0 & 0 & 4 a & 0 & 4 a^{2} b \\ I & 2 b I & b(a b+1) I & \end{array}\right|$

$=a^{10} b(a b+1)\left|\begin{array}{ccc}I & 2 b I & b(a b+1) I \\ 4 I & 4 b I & 0 \\ 0 & 4 a I & 4 a^{2} b I\end{array}\right|$

$=4^{4} a^{12} b(a b+1)\left|\begin{array}{ccc}I & 2 b I & b(a b+1) I \\ I & b I & 0 \\ 0 & I & a b I\end{array}\right|$

$=-4^{4} a^{12} b(a b+1)\left|\begin{array}{cc}0 & b I \\ I & a b I\end{array}\right|$

$=4^{4} a^{12} b^{3}(a b+1)$

where $I=I_{2}$ is the identity matrix. Hence $\Delta\left(f_{2}\right)=4^{4} a^{9} b^{3}(a b+1)$.

Now for

$f_{3}(x)=a^{7} x^{8}+4 a^{6} b x^{6}+2 a^{4} b(3 a b+1) x^{4}+4 a^{3} b^{2}(a b+1) x^{2}+b\left((a b)^{3}+2(a b)^{2}\right.$

$+a b+1)$

we write $\Gamma=(a b)^{3}+2(a b)^{2}+a b+1$ for simple expression. Similar to above,

$R\left(f_{3}, f_{3}^{\prime}\right)$

$=8^{8} a^{54} b^{7} \Gamma$. 


$$
\left|\begin{array}{lllllll}
0 & b I & b(3 a b+1) I & 3 b^{2}(a b+1) I & \Gamma I & 0 & 0 \\
0 & a I & 4 a^{2} b I & 2 a b(3 a b+1) I & 4 a^{3} b(a b+1) I & \Gamma I & 0 \\
0 & 0 & a^{3} I & 4 a^{3} b I & 2 a^{4}(3 a b+1) I & 4 a^{3} b(a b+1) I & \Gamma I \\
I & 3 b I & b(3 a b+1) I & b^{2}(a b+1) I & 0 & 0 & 0 \\
0 & I & 3 a b I & b(3 a b+1) I & a^{2} b(a b+1) I & 0 & 0 \\
0 & 0 & I & 3 b I & a(3 a b+1) I & (a b+1) I & 0 \\
0 & 0 & 0 & a I & 3 a^{3} I & a(3 a b+1) I & (a b+1) I
\end{array}\right|
$$

for the identity matrix $I=I_{2}$. Continuing these long procedure, we finally get

$$
R\left(f_{3}, f_{3}^{\prime}\right)=8^{8} a^{56} b^{7}(a b+1)^{2}\left((a b)^{3}+2(a b)^{2}+a b+1\right),
$$

thus $\Delta\left(f_{3}\right)=8^{8} a^{49} b^{7}(a b+1)^{2}\left((a b)^{3}+2(a b)+a b+1\right)$.

We can develop this result by using Theorem 8 .

Theorem 16. Let $f(x)=a x^{2}+b$ be a binomial quadratic polynomial. Then $\Delta\left(f_{n}\right)=2^{n \cdot 2^{n}} a^{\left(2^{n}-1\right)^{2}} \prod_{i=1}^{n} f_{i}(0)^{2^{n-i}}$ for any $n>1$.

Proof. We first calculate the resultants $R\left(f_{n}, f_{n}^{\prime}\right)$. Note that $R\left(f, f^{\prime}\right)=$ $2^{2} a^{2} b=2^{2} a^{2} f_{1}(0)$, so $\Delta(f)=-a^{-1} R\left(f, f^{\prime}\right)=-2^{2} a f(0)$. Then

$$
\begin{aligned}
R\left(f_{2}, f_{2}^{\prime}\right) & =R\left(f \circ f,\left(f^{\prime} \circ f\right) f^{\prime}\right)=R\left(f \circ f, f^{\prime} \circ f\right) R\left(f \circ f, f^{\prime}\right) \\
& =\left(a^{2} R\left(f, f^{\prime}\right)\right)^{2} \cdot(-1)^{2^{2}}(2 a)^{2^{2}} \prod_{f^{\prime}(\beta)=0}(f \circ f)(\beta) \\
& =\left(a^{2} \cdot 2^{2} a^{2} f_{1}(0)\right)^{2} 2^{2^{2}} a^{2^{2}} f_{2}(0)=2^{2 \cdot 2^{2}} a^{2^{2}\left(2^{2}-1\right)} f_{1}(0)^{2} f_{2}(0)
\end{aligned}
$$

since 0 is the only root for $f^{\prime}(x)=0$. Similarly

$$
\begin{aligned}
R\left(f_{4}, f_{4}^{\prime}\right) & =\left(a^{2^{3}\left(2^{3}-1\right)} R\left(f_{3}, f_{3}^{\prime}\right)\right)^{2} \cdot(-1)^{2^{4}}(2 a)^{2^{4}} \prod_{f^{\prime}(\beta)=0}\left(f_{3} \circ f\right)(\beta) \\
& =\left(a^{2^{3}\left(2^{3}-1\right)} 2^{3 \cdot 2^{3}} a^{2^{3}\left(2^{3}-1\right)} f_{1}(0)^{2^{2}} f_{2}(0)^{2} f_{3}(0)\right)^{2}(2 a)^{2^{4}} f_{4}(0) \\
& =2^{4 \cdot 2^{4}} a^{2^{4}\left(2^{4}-1\right)} f_{1}(0)^{2^{3}} f_{2}(0)^{2^{2}} f_{3}(0)^{2} f_{4}(0) .
\end{aligned}
$$

Thus the mathematical induction yields that, for $n>1$

$$
R\left(f_{n}, f_{n}^{\prime}\right)=2^{n \cdot 2^{n}} a^{2^{n}\left(2^{n}-1\right)} f_{1}(0)^{2^{n-1}} f_{2}(0)^{2^{n-2}} \cdots f_{n-2}(0)^{2^{2}} f_{n-1}(0)^{2} f_{n}(0)
$$




$$
=2^{n \cdot 2^{n}} a^{2^{n}\left(2^{n}-1\right)} \prod_{i=1}^{n} f_{i}(0)^{2^{n-i}} .
$$

Now for the discriminants, we have

$$
\begin{aligned}
\Delta\left(f_{2}\right) & =a^{-\left(2^{2}-1\right)} R\left(f_{2}, f_{2}^{\prime}\right)=a^{-\left(2^{2}-1\right)} \cdot 2^{2 \cdot 2^{2}} a^{2^{2}\left(2^{2}-1\right)} f_{1}(0)^{2} f_{2}(0) \\
& =2^{2 \cdot 2^{2}} a^{\left(2^{2}-1\right)^{2}} f_{1}(0)^{2} f_{2}(0) \\
\Delta\left(f_{3}\right) & =a^{-\left(2^{3}-1\right)} \cdot 2^{3 \cdot 2^{3}} a^{2^{3}\left(2^{3}-1\right)} f_{1}(0)^{2^{2}} f_{2}(0)^{2} f_{3}(0) \\
& =2^{3 \cdot 2^{3}} a^{\left(2^{3}-1\right)^{2}} f_{1}(0)^{2^{2}} f_{2}(0)^{2} f_{3}(0) \\
\cdots & \\
\Delta\left(f_{n}\right) & =2^{n \cdot 2^{n}} a^{\left(2^{n}-1\right)^{2}} f_{1}(0)^{2^{n-1}} f_{2}(0)^{2^{n-2}} \cdots f_{n-2}(0)^{2^{2}} f_{n-1}(0)^{2} f_{n}(0) \\
& =2^{n \cdot 2^{n}} a^{\left(2^{n}-1\right)^{2}} \prod_{i=1}^{n} f_{i}(0)^{2^{n-i}}
\end{aligned}
$$

The constant term $f_{i}(0)$ can be computed explicitly: if we write $X=$ $a b$ then

$$
\begin{aligned}
& f_{2}(0)=f(f(0))=f(b)=b(a b+1)=b(X+1), \\
& f_{3}(0)=f\left(f_{2}(0)\right)=a(b(X+1))^{2}+b=b\left(X^{3}+2 X^{2}+X+1\right), \\
& f_{4}(0)=b\left(X^{7}+4 X^{6}+6 X^{5}+6 X^{4}+5 X^{3}+2 X^{2}+X+1\right) .
\end{aligned}
$$

These values can be substituted in Theorem 16 in order to have $\Delta\left(f_{i}\right)$ and $R\left(f_{i}, f_{i}^{\prime}\right)$ precisely. And we can have more interesting formulae from the monic binomial polynomial $f(x)=x^{2}+b$, where this polynomial was treated in [4] for the prime divisors of the iteration $f_{n}(x)$.

Corollary 17. Let $f(x)=x^{2}+b$ be a binomial polynomial. Then

(1) $\Delta\left(f_{n}\right)=2^{2^{n}} f_{n}(0) \Delta\left(f_{n-1}\right)^{2}$ for any $n>1$. And $R\left(f_{n+1}, f_{1}\right)=$ $f_{n}(0)^{2}$.

(2) $R\left(f_{n+t}, f_{n}\right)=f_{t}(0)^{2^{n}}=R\left(f_{n-k+t}, f_{n-k}\right)^{2^{k}}$ for any $t>0$. 
Proof. Due to Theorem 16 we have

$$
\begin{aligned}
\Delta\left(f_{n}\right) & =2^{2^{n}} \cdot 2^{(n-1) 2^{n}} \prod_{i=1}^{n-1} f_{i}(0)^{2^{n-i}} \cdot f_{n}(0) \\
& =2^{2^{n}} \cdot\left(2^{(n-1) 2^{n-1}}\right)^{2} \prod_{i=1}^{n-1}\left(f_{i}(0)^{2^{n-i-1}}\right)^{2} \cdot f_{n}(0) \\
& =2^{2^{n}}\left(2^{(n-1) 2^{n-1}} \prod_{i=1}^{n-1} f_{i}(0)^{2^{n-i-1}}\right)^{2} f_{n}(0)=2^{2^{n}} f_{n}(0) \Delta\left(f_{n-1}\right)^{2} .
\end{aligned}
$$

Let $I$ denote the identity map. Then

$$
R\left(f_{n+1}, f\right)=R\left(f_{n} \circ f, I \circ f\right)=R\left(f_{n}, I\right)^{2}=\prod_{I(\beta)=0} f_{n}(\beta)^{2}=f_{n}(0)^{2},
$$

because $I$ has unique root 0 . Similarly it follows that

$$
\begin{aligned}
& R\left(f_{n+1}, f_{n}\right)=R\left(f \circ f_{n}, I \circ f_{n}\right)=R(f, I)^{2^{n}}=\prod_{I(\beta)=0} f(\beta)^{2^{n}}=f(0)^{2^{n}} \\
& R\left(f_{n-k+1}, f_{n-k}\right)=R\left(f \circ f_{n-k}, I \circ f_{n-k}\right)=R(f, I)^{2^{n-k}}=f(0)^{2^{n-k}} \\
& R\left(f_{n+2}, f_{n}\right)=R\left(f_{2} \circ f_{n}, I \circ f_{n}\right)=R\left(f_{2}, I\right)^{2^{n}}=f_{2}(0)^{2^{n}} .
\end{aligned}
$$

In particular

$$
\begin{aligned}
& R\left(f_{5}, f_{4}\right)=R\left(f_{4}, f_{3}\right)^{2}=R\left(f_{3}, f_{2}\right)^{4}=R\left(f_{2}, f_{1}\right)^{8} \\
& R\left(f_{4}, f_{1}\right)=f_{3}(0)^{2}, R\left(f_{5}, f_{2}\right)=f_{3}(0)^{4}, \cdots, R\left(f_{n+3}, f_{n}\right)=f_{3}(0)^{2^{n}},
\end{aligned}
$$

hence

$$
\begin{aligned}
R\left(f_{n+1}, f_{n}\right) & =f_{1}(0)^{2^{n}}=R\left(f_{n-k+1}, f_{n-k}\right)^{2^{k}}, \\
R\left(f_{n+2}, f_{n}\right) & =f_{2}(0)^{2^{n}}=R\left(f_{n-k+2}, f_{n-k}\right)^{2^{k}}, \\
R\left(f_{n+2}, f_{n}\right) & =R\left(f_{n+1}, f_{n-1}\right)^{2}=R\left(f_{n}, f_{n-2}\right)^{2^{2}}=\cdots \\
& =R\left(f_{n-k+2}, f_{n-k}\right)^{2^{k}}, \\
R\left(f_{n+1}, f_{n}\right) & =R\left(f_{n}, f_{n-1}\right)^{2}=R\left(f_{n-1}, f_{n-2}\right)^{2^{2}}=\cdots \\
& =R\left(f_{n-k+1}, f_{n-k}\right)^{2^{k}} .
\end{aligned}
$$

When $b= \pm 1$ we have more interesting results immediately. 
Corollary 18. Let $f(x)=x^{2}+1$ and $g(x)=x^{2}-1$. Then

(1) $\Delta(f)=-4$ and $\Delta\left(f_{n+1}\right)=2^{\operatorname{deg} f_{n+1}} \Delta\left(f_{n}\right)^{2} f_{n+1}(0)$ for all $n>0$.

(2) $R\left(f_{n+k}, f_{n}\right)=f_{k}(0)^{2^{n}}$. And $\Delta(g)=4$ and $\Delta\left(g_{n}\right)=0$ for all $n>1$.

(3) $R\left(g_{n+1}, g_{1}\right)=g_{n}(0)^{2}$ and $R\left(g_{n}, g_{k}\right)= \begin{cases}1 & \text { if } n-k \text { odd } \\ 0 & \text { if } n-k \text { even }\end{cases}$

\section{Proof. Obviously}

$$
\begin{aligned}
\Delta\left(f_{n+1}\right) & =R\left(f_{n} \circ f, f_{n}^{\prime} \circ f\right) R\left(f_{n} \circ f, f^{\prime}\right)=R\left(f_{n}, f_{n}^{\prime}\right)^{2} R\left(f_{n} \circ f, 2 x\right) \\
& =\Delta\left(f_{n}\right)^{2} R\left(f_{n+1}, 2\right) R\left(f_{n+1}, x\right)=\Delta\left(f_{n}\right)^{2} 2^{\operatorname{deg} f_{n+1}} f_{n+1}(0) .
\end{aligned}
$$

Moreover since $g_{n}(0)=-1$ if $n$ is odd and $g_{n}(0)=0$ otherwise, the rest follows immediately.

In particular $R\left(f_{n+1}, f_{n}\right)=1, R\left(f_{n+2}, f_{n}\right)=2^{2^{n}}, R\left(f_{n+3}, f_{n}\right)=5^{2^{n}}$ and $R\left(f_{n+4}, f_{n}\right)=26^{2^{n}}$.

\section{References}

[1] H. Cohen. Resultants and Discriminants. A Course in Computational Algebraic Number Theory. New York, Springer Verlag, (1993) 119-123.

[2] D. Cox, J. Little, D. OShea. Using Algebraic Geometry. New York, Springer Verlag, 1998.

[3] R.N. Goldman, T. Sederberg, D. Anderson. Vector elimination: a technique for the implicitization, inversion and intersection of planar parametric rational polynomial curves. Comput. Aided Geom. Des., 1, (1984) 327-356.

[4] R. Jones, The density of prime divisors in the arithmetic dynamics of quadratic polynomilas, J. London Math. Soc. 78 (2), (2008) 523-544.

[5] J.T. Kajiya. Ray tracing parametric patches. Proceedings of SIGGRAPH (1982) $245-254$.

[6] D. Manocha, J. Canny. Multipolynomial resultant algorithms. J. Symbolic Computation, 15(2), (1993) 99-122.

[7] J.H. McKay, S.S. Wang, A chain rule for the resultant of two polynomials, Arch. Math., 53, (1989) 347-351.

[8] R. W. K. Odoni, The Galois theory of iterates and composites of polynomials, Proc. London Math. Soc., 51 (1985) 385-414.

[9] R. W. K. Odoni, Realising wreath products of cyclic groups as Galois groups, Mathematika, 35, (1988) 101-113.

[10] J.R. Silvester. Determinants of block matrices. Math. Gazette, 84 (501), Nov. (2000) 460-467. 
[11] B.L.van der Waerden, Algebra, Vol 1. New York, 1970. (translat from German edition, 1966)

[12] S.H. Weintraub, Galois Theory, New York, Springer Verlag, 2005.

\section{Eunmi Choi}

Dept. Math.

HanNam University,

Daejon, Korea,

e-mail : emc@hnu.kr 